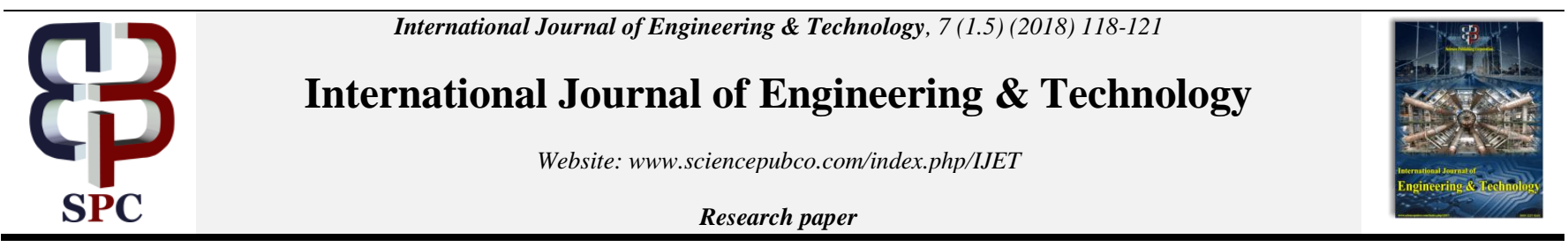

\title{
Multi-hope relay based high capacity energy efficient model for ultra wideband communication system
}

\author{
B.Rajanna ${ }^{1 *}$, P. Chandrashrkar ${ }^{2}$, K. KishanRao ${ }^{3}$ \\ ${ }^{1}$ Research scholar, Department of electronics and communication engineering \\ Vel Tech Dr.RR\&Dr SR Technical University Chennai \\ ${ }^{2}$ Asso.prof EEE HOD, Vel Tech Dr RR \&Dr SR Technical University Chennai \\ ${ }^{3}$ Senior IEEE member, Professor of ECE \& Director, \\ Sreenidhi Institute of Science \&Technlogy, Hyderabad-501301(T.S.) \\ *Corresponding author E-mail: rajannabattula@gmail.com.com
}

\begin{abstract}
Demanding large bandwidth and signal robustness over a short distance, ultra wideband communication systems plays an important role. UWB systems exhibits very low spectral density, implies that the interference to narrowband systems is very small. The relay based communication has the advantages of coverage extension, range extension, increase data rates and green communication. The relay based communication with UWB frequency is used to increase the coverage area by using of relays and increasing the data rates by increasing SNR of the links using multi-hop communication. In this paper, capacity and bit error rate of relay based UWB system for indoor and outdoor channel models are analyzed. key Terms - UWB, green communication, multi-hop, capacity, bit error rate, spectral density.
\end{abstract}

\section{Introduction}

Ultra wide band communication is actively considered as an alienation for high bandwidth communication and is looked by lot of communication research groups. Because of limitations such as (i) Coverage area (ii) NLOS communication (iii)Radiation effect at their frequency, multiuser communication using relay scheme is an alternative to obtain coverage extension and obtain high data rates. Shannon theorem dictates that data rates should be maximized with channel bandwidths and signal to noise ratio. SNR will be maximized with transmitting power increased or relay based communication but the percentage of increment is very low. Therefore migration from narrow band to ultra wide band communication with frequency ranges from $2-10 \mathrm{GHz}$, increases the data rates. UWB mainly used in low power, short-range and high bandwidth communication applications i.e., indoor residential/office, Open outdoor and industrial for high bit rates. UWB signalling has a vital role in future mobile networks which uses bandwidths in excess of $3 \mathrm{GHz}$ results in following advantages:

- Position tracking and ranging

- Security

- $\quad$ Easier penetration (see-through-the-material)

- Decrease in fading effect

- Model for LOS and NLOS

There are some of the disadvantages which leads to non cooperation in implementation of system are

- Coverage area

- Penetration of UWB signals to living creatures

There are different kind of applications where the ultra wide band must be used i.e., 1) Wireless USB connection 2) With in coverage area, very high data rates for data and multimedia communication using digital pulses 3) Wireless machine to ma- chine communication in indoor office environment 4) Wireless printing 4) Accurate positioning and many more. The design of transceivers and performance evaluation of the system depends upon the channel model, frequency range and communication scheme used. There are different channel models used in UWB, Tap-delay line Rayleigh fading channel, $\Delta-\mathrm{K}$ model and Saleh Valenzuela. Modified SV model is popular to describe UWB environment by the following parameters

1)Cluster arrival time ( $\Lambda)$ 2)Ray arrival time ( $\lambda$ ) 3)Cluster decay factor $(\Gamma)$ 4)Ray decay factor $(\gamma)$ The relay based communication has the advantages of coverage extension, range extension, increase data rates and green communication. The relay based communication with UWB frequency is used to increase the coverage area by using of relays and increasing the data rates by increasing SNR of the links using multi-hop communication.

The objective of this paper is to increase the data rates and coverage/range extension of UWB communication in LOS and NLOS conditions.

The rest of the paper is organized as follows. Section II gives brief overview of UWB signaling, channel models and relay communication. Section III presents the system model and performance metrics evaluation under different scenarios. The simulation results and discussion was presented in section. The conclusion of the paper and future work was provided in sectionV.

\section{Literature Review}

Ultra wide band communication channels were modelled $[2][3][4][6]$ by various authors and can be summarized as: Amendments on Saleh-Valenzuela (SV) multi path channel model has done to model a UWB IEEE slandered i.e., IEEE 802.15.3a, also known as modified S-V model. Authors modelled the modeled modified S-V modeled as a two-dimensional augmented 
cluster process in [2].Different cases were considered for channel parameters to evaluate closed-form expression.

The performance evaluation of model [2] in terms of SNR with two different types of receivers by the theoretical analysis and treating clustered process as a two-dimensional Poisson distribution explained in [3]. The performance metrics were compared by deriving closed form expressions and by numerical analysis. [4] modeled three different UWB channel models at different band of frequencies for the model IEEE 802.15.4a. Authors modeled IEEE 802.15.4a with different environments and frequency ranges and differentiated between line of sight and Nonline of sight communication. They modeled every parameter in different environment and compared the narrow band and ultra wide band by modeling channel around $1 \mathrm{MHz}$ frequency with narrow band. Tabulated the parameters and mat lab code was given to produce the impulse response of modified $\mathrm{S}-\mathrm{V}$ channel model.

The performance of the relay system in terms of outage probability under IEEE 802.15.3a channel model has been discussed in [6]. Considered a detect-and-forward relay system with multiple antennas equipped in it. The advantages for considering multiple relay antennas, pre/post-rake receivers and relays have been illustrated. Numerical results for low outage probability by avoiding spatial correlation between the rake fingers has derived and presented the future scope.

Ultra wide band multiuser communication by using the relay scheme was discussed in [1]. Authors used a two way filter as the relay signal processing unit to eliminate the burden of user terminals in transmission and reception. A centralized pre/post rake-relay with ' $M$ ' number of transmitters and receivers was considered and the design issues of filter in relay has presented. They formulated the filter design problem and demonstrated the numerical analysis with less signal processing power and no direct link.

The cooperative relay networks for ultra wide band communication with UWB signal unique feature was discussed in [5]. Explained the advantages of integrating cooperative relay networks with UWB. Authors used profiteering algorithms for space-time focusing transmission i.e., ZF and MMSE and integrated cooperative relay scheme. With the assumption of well performance of profiteering, optimal cooperative nodes to maximize the network through was derived.

Performance evaluation of system in terms of bit error rate of various ultra-wide band multiple-input multiple-output relay systems has been presented in [7]. Authors compared and examined the relay systems and cooperative relay systems by considering dual-hop MIMO network. The channel model and measurements for line-of-sight as well as non-line-of sightin indoor environment with range considered as $4 \mathrm{~m}$ were simulated. Simulations also carried out and compared for direct link(Non relay).

The coverage extension of UWB communication [8] and narrow band communication [9] using dual-hop and multi hop modeled for high data rates. [8]considered dual-hop UWBMIMO relay system and performance was analyzed over UW Bmulti path fading. Different relays were proposed at transmitter and receiver with the knowledge of channel state information (CSI). The expression for outage probability, bit error rate and amount of fading for proposed model under high SNR condition has been derived and verified with simulations. Where as [9] discusses the coverage extension and data rate increment energy efficiently.

\section{Model Considered}

Consider a communication link between source and sink in a picocell uses the ultra-wide band frequencies. [9] concentrated on data rate enhancement and energy efficiency for narrow band communication for urban and rural environment. The paper migrates from micro-cell to pico-cell by wandering from narrow band to ultra-wide band.

\section{A. Channel model}

The nodes were deployed randomly which satisfies LOS and NLOS condition in the pico-cells. The relays were placed as discussed in [9] and [5] leads to increase the data rates and lowenergy communication. The evaluation of throughput of this model depends upon the realization of channel model. The different channel models are:

1) Rayleigh fading channel: Rayleigh fading used in non line of sight radio communication in urban environment where the signal reception done by multiple signals (say ' $N$ '). Rayleigh is preferable when more scattering of radio signal happens in the transmission. The fading of the ' $k$ th' waveform over time ' $t$ ' can be modeled by using jake's model as

$$
\begin{aligned}
& H(t, k)=2 \sqrt{2} \quad\left[\quad \sum_{t=1}^{M}\left(\cos \beta_{1}+j \sin \beta_{1}\right) \cos \left(2 \pi f_{1} t+\theta_{t, k}\right)+\right. \\
& \left.\frac{1}{\sqrt{2}}(\cos \alpha+j \sin \alpha) \cos 2 \pi f_{i} t\right]
\end{aligned}
$$

2) Rician fading channel: The reception with LOS path is more preferable compared with multi path traveled signal. The fading channel with line of sight communication modeled as Rician fading, the PDF of the channel is

$\mathrm{P}(\mathrm{x})=\frac{2(K+1)}{\sigma} \exp \left(-k-\frac{(k+1) x^{2}}{\sigma}\right) k_{0}\left(\sqrt[2]{\frac{K(K+1)}{\sigma}} x\right)$

where $\mathrm{K} 0$ is the 0th ordered modified Bessel's function of first kind.

The above two models are preferred in cellular/narrow band communication. But the analyzing of UWB communication is not possible with normal fading channels, where it has to analyse by using different fading channels at different conditions as follows.

3) Modified S-V model: The effects arises for UWB channels compared to conventional wireless channel are to be considered To model a new channel with different parameters effects the fading. The central limit theorem is no longer applicable because the overlap of multi path components and fading statistics are no longer Rayleigh and Rician. The impulse response of modified SV model given by [2][3][4][6] is

$H_{i}(x)=X_{i} \sum_{l=0}^{L} \sum_{k=0}^{K} \alpha_{k, l}^{i} \delta\left(x-T_{l}^{i}-\tau_{k, l}^{i}\right)$

\section{B. System model}

The relay based communication with dual hop and multihop communication is used ti increase the pico-cell coverage to some extent. There are two different channel models in any wireless networks i.e., line-of-sight (LOS) and Non-lineof- sight (NLOS). The ranges of these models are depends upon the frequency of operation, environment effects (rual/urban) and transceiver design etc.,. The communication range of UWB for LOS is about $4 \mathrm{~m}$ and for NLOS it is $4-10 \mathrm{~m}$.

The relay based UWB communication is mainly used in NLOS condition to make it LOS, to increase coverage and data rates. The performance evaluation of dual hop and multi-hop system is as follows: 1) Dual hop: The system mode in [9] considered for UWB with ' $\mathrm{D}=80 \mathrm{~m}$ ', communication range of relay ' $C_{r}=40 \mathrm{~m}$ ' and $\mathrm{AF}$ relay with relay gain $\mathrm{G} 2=\frac{1}{H_{S R}^{2}}$, upper bound of $\mathrm{AF}$ relay gives better performance. From the above considerations the system parameters leads to

$$
\gamma_{D H}=\frac{\gamma_{1} \gamma_{2}}{\gamma_{1}+\gamma_{2}+\frac{1}{N_{0}^{2}} \sum_{j \neq i} \frac{p\left(e_{j}\right)}{d_{j R}^{\alpha}}}
$$

Where $\mathrm{i}$ is SNR of ith relay link, $p\left(e_{j}\right)$ and $d_{j R}^{\alpha}$ are received power and distance from interference source ' $\mathrm{j}$ ' to relay ' $\mathrm{R}$ ' and $\alpha$ is path loss exponent.

2) Multi-hop: The extension of dual hop to multi-hop leads to increase coverage, increase data rates and also increase in energy 
and interference because of large bandwidth communication. Let us consider the placement [9] and with above mentioned parameters in dual hop, the system parameters calculated as

$\gamma_{M H}=\frac{\gamma_{1} \gamma_{2} \gamma_{3} \ldots \gamma_{N}}{\gamma_{1}+\gamma_{2}+\cdots \ldots+\gamma_{N}+\sum_{r=1}^{N} \sum_{j \neq i} \frac{p\left(e_{j r}\right)}{d_{j R r}^{\alpha}}}$

Where $p\left(e_{j r}\right)$ and $d_{j R r}^{\alpha}$ are power received and distance from the interference siurce ' $\mathrm{j}$ ' to relay ' $\mathrm{Rr}$ '. From eqns (5) and (6), the SNR of ' $N$ ' multi-hop interference free system can be writtens as harmonic mean of individual SNR's of 'N' hops i.e.,

$\gamma_{\mathrm{N}}=\frac{\mathrm{H}\left(\gamma_{1} \gamma_{2} \gamma_{3 \ldots \gamma_{\mathrm{N}}}\right)}{\mathrm{N}}$

The capacity equation form Shanon theorem and the energy efficient model from [9] are givind the following equations.

$\mathrm{C}=\mathrm{B} * \log _{2}\left(1+\frac{\mathrm{S}}{\mathrm{N}}\right)$

And

$\mathrm{N}_{\mathrm{opt}}=\frac{\mathrm{D}}{\mathrm{d}_{0}} \sqrt[\alpha]{\frac{\mathrm{p}_{\mathrm{S}}(\alpha-1)}{\mathrm{P}_{\mathrm{R}}}}$

where $\mathrm{N}_{\text {opt }}$ is optimal relays for green communication, $\mathrm{P}_{\mathrm{R}}$ reception cost of relay and PS transmission cost. The performance evaluation of system models in terms of capacity (data dates) and energy (green) for ultra wide-band by using the equations (1)-(7) are simulated and comparisons discussed in following chapters.

\section{Results and discussion}

The simulations are carried out by using matlab. The parameters given in Table I and II are considered for simulations purpose. TABLE I: Specifications Considered for UWB system

\begin{tabular}{|l|l|}
\hline Parameter & values \\
\hline Channel bandwidth & $3 \mathrm{GHz}$ \\
\hline S-D distance & $40-80 \mathrm{~m}$ \\
\hline Channel & AWGN \\
\hline Hops considered & 2 \\
\hline Communication range & $40 \mathrm{~m}$ \\
\hline Path loss exponent $(\alpha)$ & 2 \\
\hline $\begin{array}{l}T_{X} \text { power } \\
\text { Relay } \\
\text { Model }\end{array}$ & $\begin{array}{l}1 \text { unit } \\
\text { AF relay } \\
\text { ayleigh fading Rician fading Modifeied SV model }\end{array}$ \\
\hline Enssemble average & 1000 \\
\hline
\end{tabular}

The Table I gives the considerations for the system to simulate in the mat lab. The parameters of the channel for ultra wide band (UWB) communication in indoor and outdoor environment by considering LOS and NLOS conditions were tabulated in Table II.

\begin{tabular}{|l|l|l|l|l|} 
Parameter & $\begin{array}{l}\text { Model-1 } \\
\text { Arrival time } \\
\text { of cluster }\end{array}$ & $\begin{array}{l}\text { Model-2 } \\
0.0233\end{array}$ & $\begin{array}{l}\text { Model-3 } \\
0.4\end{array}$ & $\begin{array}{l}\text { Model-4 } \\
0.067\end{array}$ \\
\hline $\begin{array}{l}\text { Arrival time } \\
\text { of ray }\end{array}$ & 2.5 & 0.5 & 2.1 & 2.1 \\
\hline $\begin{array}{l}\text { Decay factor } \\
\text { of cluster }\end{array}$ & 7.1 & 6.7 & 14 & 24 \\
\hline $\begin{array}{l}\text { Decay factor } \\
\text { of ray }\end{array}$ & 4.3 & 3.4 & 7.9 & 12 \\
\hline
\end{tabular}

The Figure 1 shows that capacity of relay based communication at UWB frequency not only depends upon the relay placement but also depends on channel model. Four models (Model-1 to Model4) with different decay and arrival factors are considered by assuming the channel models as:

- Indoor with LOS (Model-1)

- $\quad$ Outdooe with LOS (Model-2)

- Indoor with NLOS (Model-3)

- $\quad$ Outdoor with NLOS (Model-4)

The capacity variations with respect to relay placement for different channel models was presented in Figure 1. The figure shows an increase in data rates for UWB system with relay.

Dual-hop relay capacity with respect to relay placement

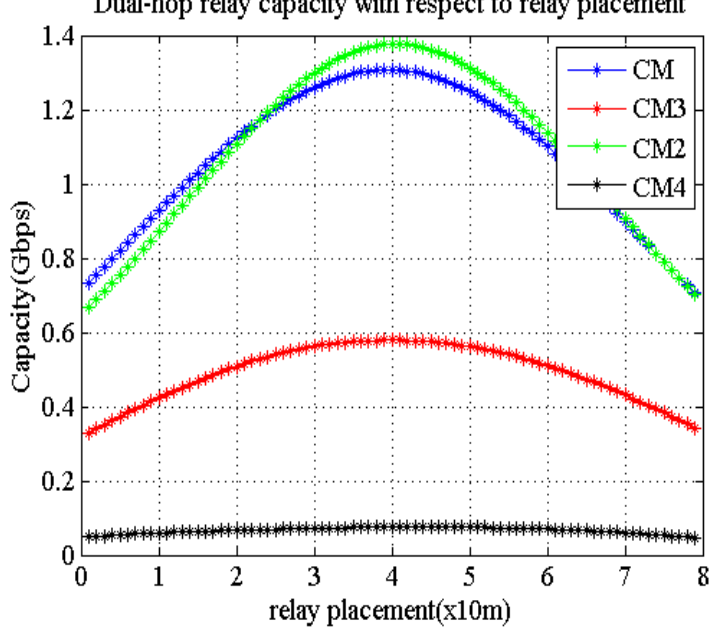

Fig. 1: Data rate increment of relay based UWB system.

placed equidistance to source and destination. The capacity increment is approximately double if the user is LOS with source in indoor and outdoor environment, as shown by green and blue in Figure 1. There was less increase in capacity under NLOS condition in indoor environment (red line) where as increment is not possible in outdoor environment with NLOS condition.

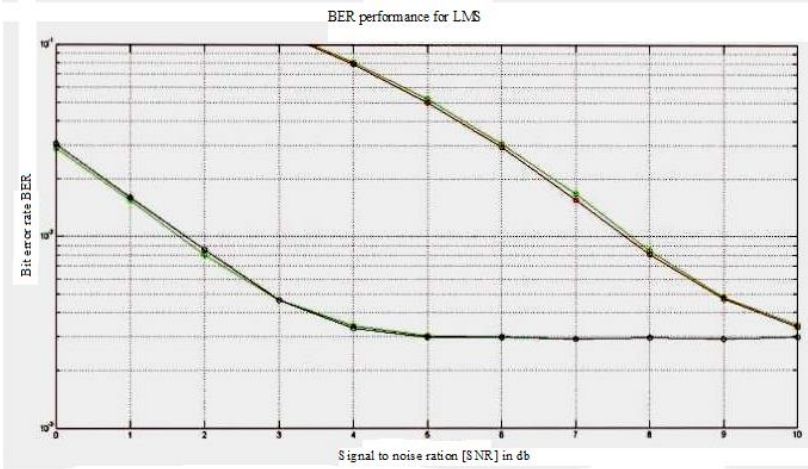

Fig.2: Bit error rate comparison for narrowband and ultra wideband

The bit error rate comparison of direct link with respect to dual hop relay link was presented in Figure 2. There is an effect on bit error rate compared to direct link in UWB but there is less effect on it when narrowband frequencies were considered with same specifications under both 1) LOS and 2) NLOS conditions. The Figure 2 shows the comparison of BER for NLOS communication, narrowband communication with Rayleigh fading Ultra wideband communication with Modified SV model.

\section{Conclusion}

From the results it can be concluded that the relay based UWB communication enhances the data rates excluding for outdoor NLOS channel model. The percentage of increment of capacity for UWB LOS communication is more compared to UWB NLOS 
communication. There is less variation of bit error rate for UWB when compared to the narrowband.

\section{References}

[1] Z. Ahmadian, L. Lampe and J. Mietzner, "Multiuser two-way filter-andforward relaying for ultra-wideband communications," IEEE 80th Vehicular Technology Conference (VTC2014-Fall), Vancouver, BC, 2014, pp.1-6.

[2] Gubner, John A. and Kei Hao, "The IEEE 802.15. 3a UWB channel model as a two-dimensional augmented cluster process.' submitted for publication 2006.

[3] K. Hao and J. A. Gubner, "Theoretical performance analysis of the IEEE 802.15.3a UWB channel model," IEEE Global Telecommunications Conference, Washington, DC, 2007, pp 1770-1774.

[4] Molisch, Andreas F., et al. "IEEE 802.15. 4a channel model-final report."

[5] Y. Tian and C. Yang, "Space-time focusing transmission in ultra wideband cooperative relay networks," IEEE International Conference on Ultra-Wideband, Vancouver, BC, 2009, pp. 353 358.

[6] K. Maichalernnukul, T. Kaiser and F. Zheng, "Performance investigation of a UWB relay system using multiple relays with multiple antennas in IEEE 802.15.3a channel," Vehicular Technology Conference, 2009. VTC Spring 2009. IEEE 69th, Barcelona, 2009, pp. 1-6.

[7] K. Maichalernnukul, T. K. Nguyen, F. Zheng and T. Kaiser "Performance of UWB MIMO relay systems in real UWB channels," FortyFourth Asilomar Conference on Signals, Systems and Computers, PacificGrove, CA, 2010, pp. 526-530.

[8] K. Maichalernnukul, F. Zheng and T. Kaiser, "Design and performance of dual-hop MIMO UWB transmissions," IEEE Transactions on Vehicular Technology, vol. 59, no. 6, pp. 29062920, July 2010.

[9] T.Padmapriya and V.Saminadan, "Utility based Vertical Handoff Decision Model for LTE-A networks", International Journal of Computer Science and Information Security, ISSN 1947-5500, vol.14, no.11, November 2016

[10] S.V.Manikanthan and D.Sugandhi “ Interference Alignment Techniques For Mimo Multicell Based On Relay Interference Broadcast Channel " International Journal of Emerging Technology in Computer Science \& Electronics (IJETCSE) ISSN: 0976-1353 Volume- 7 ,Issue 1 -MARCH 2014.

[11] Rajesh, M., and J. M. Gnanasekar. \&quot;Congestion Control Using Aodv Protocol Scheme For Wireless Ad-Hoc Network.\&quot; Advances in Computer Science and Engineering 16.1/2 (2016): 19 